\title{
Proyecto reflexivo de co-evaluación y auto-evaluación formativa utilizando la técnica del Puzzle de Aronson dentro del Aprendizaje Cooperativo
}

\author{
Ahís Adell, M.* \\ *Licenciada en Ciencias de la Actividad Física y el Deporte y Graduada en Fisioterapia. E-mail: \\ mahisadell@gmail.com

\section{Abstract} \\ This study aims to analyze the teaching-learning process of the Physical Education (PE), by comparing \\ the perceptions of students in first years of high school and their two respective teachers. This is a case \\ study, based on qualitative research techniques: semi-structured interviews, participant and general \\ observation and a research cooperative innovation based on the concept of Aronson Puzzle. The results \\ show the existence of a gap between teory defining the objective of the subject and application of this in \\ the classroom. This is conditioned by limitations of the educational context, but also by professional \\ constraints which do not always have consciousness.
}

Keywords: Physical Education, cooperative learning, evaluation, innovation, competencies, high school, Aronson Puzzle

\section{Resumen}

El presente estudio tiene por objetivo la evaluación del proceso de enseñanza-aprendizaje de EF, mediante la comparación de las percepciones del alumnado de dos cursos de primero de Bachillerato y sus dos respectivos profesores/as. Se trata de un estudio de caso, basado en técnicas de investigación cualitativas: entrevistas semiestructuradas, observación participante y general y una innovación competencial cooperativa basada en el Puzzle de Aronson. Los resultados muestran la existencia de una distancia entre la definición teórica del objetivo de la asignatura y la aplicación de esta en el aula. Este hecho se ve condicionado por limitaciones del contexto educativo, pero también por limitaciones profesionales de las cuáles no siempre se tiene consciencia

Palabras clave: Educación Física, aprendizaje cooperativo, evaluación, innovación, competencias, bachillerato, Puzzle de Aronson.

\section{Introducción}

\section{Aproximación al concepto de evaluación}

El presente trabajo se centra en la evaluación del proceso de enseñanza-aprendizaje mediante la comparación de las percepciones que tienen de la Educación Física (EF) el alumnado y el profesorado, protagonistas directos de este proceso. Tal y como afirmaba Blázquez (2017) la evaluación constituye un proceso compartido que, en función de unos criterios, obtiene evidencias de aprendizaje, tanto del transcurso como del producto, para reflexionar y formular juicios sobre los cambios y resultados, y con el fin de poder tomar las decisiones más adecuadas que ayuden al alumnado a ser más responsable y autónomo en cualquier decisión de la vida. De esta definición se extrae que los resultados de la evaluación sirven al profesorado para tomar medidas y mejorar el proceso de enseñanza-aprendizaje. Y además, Blázquez (2017) pone especial énfasis en el poder de la evaluación como motor de aprendizaje, ya que desde la perspectiva de los alumnos/as supone una tarea de reflexión que favorece el desarrollo del sentido crítico y su autonomía, y para los profesores/as constituye una herramienta que no solo les da información sino que les hace tomar consciencia real acerca de su ejercicio profesional y motivar su actualización. 
Atendiendo al marco legislativo de educación actual, el Real Decreto 1105/2014 en el punto 4 del artículo 20, nos dice que los profesores/as evaluarán tanto los aprendizajes del alumnado, como los procesos de enseñanza y su propia práctica docente. Este trabajo se centrará en las dos últimas variables, dado que la evaluación del aprendizaje del alumnado es un proceso más normalizado por su necesaria asociación y posterior justificación del proceso de calificación.

Para ello podemos utilizar diferentes técnicas en función del agente de evaluación que interviene (López Pastor, 2006; González Rivera y Campos Izquierdo, 2014; Blázquez, 2017). En este trabajo nos interesa destacar la auto-evaluación (realizada por el profesorado) y la co-evaluación (realizada por los alumnos/as).

En esta misma línea, Jackson (1992, citado por Gutiérrez \& Pilsa, 2006) resaltaba la importancia del análisis de las actitudes de los discentes (coevaluación), por la repercusión que estas tienen, tanto en la efectividad de los docentes, como en el diseño de programas educativos más adaptados a sus necesidades. En este sentido, existen numerosos trabajos que se centran en la percepción que tienen los alumnos/as respecto a la $\mathrm{EF}$, abordados desde perspectivas diversas:

-el comportamiento del profesorado y los contenidos del programa de EF (Gutiérrez \& Pilsa, 2006)

-la autonomía de los discentes en función del feed-back proporcionado por el profesor/a (Huéscar \& Moreno, 2012); el interés del alumnado hacia la asignatura (Moreno \& Hellín, 2007)

-la relación entre el clima motivacional y la capacidad percibida de rendimiento de los alumnos/as (Parish \& Treasure, 2003)

-la relación existente entre la percepción de utilidad de las clases de EF y el nivel de actividad físicodeportiva habitual (Rodríguez, García, Sánchez \& López, 2013)

-la opinión abierta sobre cualquier aspecto relacionado con las clases de EF (Blandez, 2001)

-la relación entre clima motivacional, motivación intrínseca, comportamiento del alumnado y rendimiento académico en la asignatura (Gutiérrez \& López, 2012).

Por otro lado, la interpretación que hacen los docentes (auto-evaluación) acerca de la asignatura de EF también ha despertado el interés de diferentes autores, y al igual que se ha hecho con los alumnos/as, estos trabajos se han abordado de la misma manera, desde variadas categorías de análisis: el concepto de actividad física y las estrategias para mejorar la experiencia de los estudiantes en EF y aumentar sus niveles de actividad física durante las clases (Bennie \& Langan, 2014); el comportamiento de los estudiantes desde la visión docente (Hodges, Cothran \& Regualos, 2006); o la importancia de la experiencia docente, investigando acerca de las dificultades que presentan profesores de EF noveles a la hora de ejercer la profesión (Shoval, Erlich \& Fejgin, 2010).

\section{Metodología competencial: Aprendizaje Cooperativo (AC) y Puzzle de Aronson}

Con la Ley Orgánica 2/2006, de 3 de mayo, de Educación (LOE) y la Ley Orgánica 8/2013, de 9 de diciembre, para la Mejora de la Calidad Educativa (LOMCE) aparecen las competencias como un nuevo elemento curricular a tener en cuenta. Siguiendo a Abraham Lincoln, 1862, que hacía referencia a que no se puede educar a las generaciones del futuro con herramientas que forman parte del pasado, destacamos la siguiente cita:

"los dogmas del pasado silencioso son inadecuados para el presente tormentoso. La ocasión está llena de dificultades, y debemos levantarnos con la ocasión. Como nuestro caso es nuevo, debemos pensar nuevamente y actuar de nuevo".

$\mathrm{Y}$ es que las competencias surgen en parte, para mejorar o complementar las limitaciones de la enseñanza tradicional, basada en aprendizajes memorísticos con menor aplicación práctica o transferencia a la vida cotidiana. Una de las aportaciones que ha generado el enfoque por competencias es la adopción de nuevas metodologías. Así pues, en los últimos años, diferentes autores identifican el Aprendizaje Cooperativo (AC) como uno de los modelos pedagógicos competenciales emergentes aplicados a la EF (Metzler, 2011; Dyson y Casey, 2012) debido a su capacidad para promover que el alumnado alcance los objetivos propios del área mediante un proceso estructurado que interrelaciona enseñanza, aprendizaje, 
contenido y contexto. La enseñanza a través de proyectos con una orientación cooperativa se erige pues, como uno de los referentes para la adquisición de competencias, persiguiendo la idea de educar para cooperar y educar cooperando.

$\mathrm{El} \mathrm{AC}$ es un modelo pedagógico en el que los alumnos/as trabajan juntos en pequeños grupos, generalmente heterogéneos, para maximizar su propio aprendizaje y también el del resto de compañeros/as. La co-responsabilidad en el aprendizaje es la característica sustancial de la definición y lo que diferencia al AC del mero trabajo grupal.

Existen diferentes técnicas para llevar a cabo el AC (Velázquez, 2010; Ruiz Omeñaca, 2017; González García y Fernández-Río, 2017), como el marcador colectivo, Piensa- Comparte-Actúa, los Desafíos-Retos cooperativos, la Invención-Diseño de juegos/tareas, el Puzzle de Aronson (1978)-Puzzle cooperativo, etc.

Este trabajo se centra en esta última técnica, el Puzzle de Aronson, basado en un método de doble agrupamiento: un equipo de aprendizaje (el grupo de origen) y un equipo de expertos (que cuenta con la información objeto de aprendizaje). Se suele utilizar para el aprendizaje de las técnicas de los diferentes deportes, pero en este caso, lo utilizaremos como herramienta de evaluación formativa y cooperativa (aludiendo a la doble función del proceso de evaluación expuesta en el apartado anterior). Esta idea aprovecha las características descritas en el párrafo anterior acerca del $\mathrm{AC}$ (pequeños grupos, heterogéneos, co-responsabilidad, educar para cooperar, educar cooperando...), para aplicarlas al proceso de evaluación con doble finalidad, como registro de información y posterior reajuste del proceso de enseñanza-aprendizaje, y como evaluación formativa para cooperar y evaluar cooperando. Se detallará su aplicación en el epígrafe de metodología cuando se habla de los instrumentos utilizados.

\section{Objetivos generales y específicos}

Atendiendo a todo lo expuesto, el objetivo de la presente investigación es aproximarse a las clases de EF de dos cursos de $1^{\circ}$ de Bachillerato de un centro educativo público de Valencia, a través de un proyecto de evaluación cooperativa del proceso de enseñanza-aprendizaje y la práctica docente, mediante la comparación de las percepciones que tienen el profesorado (auto-evaluación) y el alumnado (coevaluación) de dichos cursos.

Con ello, de forma específica, se pretende no solamente recoger información para poder adaptar el proceso de enseñanza-aprendizaje (objetivo principal del proceso de evaluación), sino también favorecer el aprendizaje en sí mismo, tanto de alumnos/as como de profesores/as, a través de la reflexión, el sentido crítico y el conocimiento consciente. Para este propósito, se busca implementar como innovación educativa el uso de nuevas metodologías competenciales como el AC, y dentro de este, la técnica del Puzzle de Aronson no solo como forma de aprendizaje, sino también como forma de reflexión y evaluación cooperativa.

\section{Metodología}

El presente trabajo se enmarca dentro de la investigación cualitativa, en el paradigma interpretativo. Utiliza como diseño de investigación el estudio de un caso que, según Stake (1995), se define como "el estudio de la particularidad y de la complejidad de un caso singular, para llegar a comprender su actividad en circunstancias importantes".

"La distinción fundamental entre investigación cuantitativa e investigación cualitativa estriba en el tipo de conocimiento que se pretende. Aunque parezca extraño, la distinción no está relacionada directamente con la diferencia entre datos cuantitativos y datos cualitativos, sino con una diferencia entre búsqueda de causas frente a búsqueda de acontecimientos. Los investigadores cuantitativos destacan la explicación y el control; los investigadores cualitativos destacan la compresión de las complejas relaciones entre todo lo que existe" (Stake, 1995) 


\section{Participantes y descripción del contexto}

En esta investigación han participado 39 estudiantes de $1^{\circ}$ de Bachillerato del curso 2015-2016, de un centro público de la ciudad de Valencia, ubicado en un barrio con fuerte presencia de personas migrantes y de un nivel socio-económico bajo. La muestra está compuesta por alumnado de dos grupos cuya media de edad se sitúa entre los 16 y los 17 años. La distribución por curso y sexo se puede observar en la Tabla 1.

Se ha determinado como único criterio de selección, que los alumnos/as pertenecieran a los primeros cursos de Bachillerato, por la necesidad de participación de alumnado con capacidad crítica y autonomía suficiente, para aplicar uno de los instrumentos.

Tabla 1. Distribución de la muestra por curso y sexo

\begin{tabular}{|c|c|c|c|c|c|c|}
\hline \multirow{2}{*}{ Curso } & \multicolumn{2}{|c|}{ Hombres } & \multicolumn{2}{|c|}{ Mujeres } & \multicolumn{2}{c|}{ Total } \\
\cline { 2 - 7 } & $\mathrm{n}$ & $\%$ & $\mathrm{n}$ & $\%$ & $\mathrm{n}$ & $\%$ \\
\hline $1^{\text {o }}$ Bach. A & 5 & $13 \%$ & 13 & $33 \%$ & 18 & $46 \%$ \\
\hline $1^{\text {a }}$ Bach. B & 6 & $15 \%$ & 15 & $38 \%$ & 21 & $54 \%$ \\
\hline Total & 11 & $28 \%$ & 28 & $72 \%$ & 39 & $100 \%$ \\
\hline
\end{tabular}

Por otro lado, también han participado los dos respectivos profesores/as de EF, cuyas características se resumen en la Tabla 2.

Tabla 2. Características de los profesores/as

\begin{tabular}{|c|c|c|c|c|}
\hline & Sexo & Edad (años) & Formación & Experiencia docente (años) \\
\hline Profesora A & mujer & 52 & Lic. otra especialidad & $>15$ \\
\hline Profesor B & $\begin{array}{c}\text { hombr } \\
\text { e }\end{array}$ & 50 & Lic. especialidad EF & $>15$ \\
\hline
\end{tabular}

\section{Instrumentos y compromisos éticos}

Se llevó a cabo un trabajo de campo, aprovechando el periodo de prácticas curriculares del Máster Universitario de Profesor/a de Secundaria, desarrollado durante 2 meses en dicho centro educativo. La observación, siguiendo las posibilidades apuntadas por Wolcott (1973), consistió en:

-Observación participante, en respuesta a las demandas de las prácticas, y observación general, utilizando un registro anecdótico en un cuaderno de campo, 4 sesiones por semana durante 3 semanas.

-Entrevistas semidirigidas al profesorado implicado, entre 40 minutos y 1 hora de duración, registradas mediante grabación de voz. Ambas entrevistas se desarrollaron en el departamento de EF del centro educativo. El lugar, el día y la hora fueron elegidos por el profesorado. Con ello se buscaban buenas condiciones para obtener la suficiente confianza (rapport) y conseguir datos de calidad. En ellas, se abordaron las categorías temáticas de este trabajo, es decir, la valoración de la asignatura de EF, la valoración de su utilidad e importancia y por último la valoración del rol del profesorado.

-El último de los instrumentos de recogida de datos empleado en este estudio se basa en una técnica de innovación metodológica: la aplicación de la técnica del "Puzzle de Aronson" (Martínez \& Gómez, 2010), no únicamente como recurso de "aprendizaje cooperativo" tal y como se recomienda en la literatura al respecto (Velázquez, Fraile y López Pastor, 2014), sino como instrumento de evaluación cooperativa, aprovechando los beneficios de dicha metodología, como puede ser la interdependencia y la co-responsabilidad. En este caso, se formaron grupos de discusión más reducidos y heterogéneos, de modo que todo el alumnado tuviera la oportunidad de participar en todos los temas de debate planteados y 
ser protagonistas de las conclusiones del grupo. El procedimiento se explica gráficamente a través de la Figura 1.

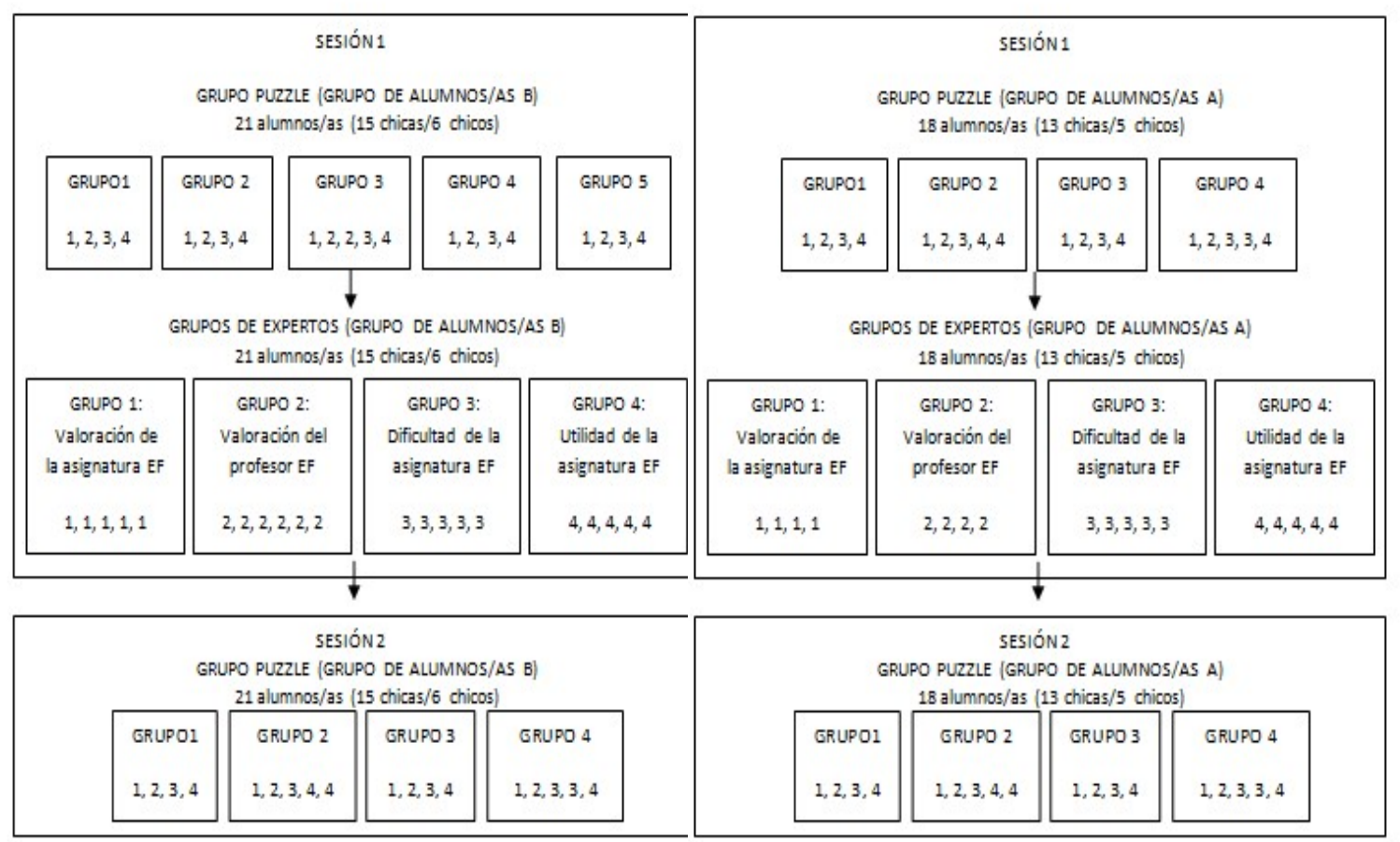

Fig. 1. Procedimiento de aplicación de la técnica del Puzzle de Aronson

Los grupos puzzle se formaron en la primera sesión en la cual, después de explicar el procedimiento, se deshicieron los mismos para formar a su vez los grupos de expertos que eran los encargados de discutir y argumentar sobre cada tema. Estas discusiones fueron grabadas y contaban con la figura de un moderador y un secretario.

En referencia a los compromisos éticos, se pidió el consentimiento informado a todos los participantes (alumnos/as y profesores/as) tanto de las observaciones, como del puzzle de Aronson y de las entrevistas. A través de él, los participantes quedaban informados de los propósitos de la investigación y sus características, así como de los riesgos y beneficios de la misma (Kvale, 2011). Para facilitar la presentación e interpretación de los resultados y mantener el anonimato de los participantes, se ha diferenciado entre Grupo A y Profesora A, y por otro lado, Grupo B y Profesor B. Por otro lado, se entregaron las transcripciones de las los instrumentos utilizados a alumnos/as y profesores/as, para su aprobación/desaprobación.

\section{Análisis de los datos}

De acuerdo con la introducción, el Real Decreto 1105/2014 en el punto 4 del artículo 20, nos dice que los profesores/as evaluarán tanto los aprendizajes del alumnado, como los procesos de enseñanza y su propia práctica docente. Dejando a un lado el aprendizaje del alumnado porque, tal y como ya se ha descrito, esta más generalizado al estar íntimamente relacionado con el proceso de calificación, se han priorizado las dos últimas variables.

Sin embargo, se han establecido categorías amplias de evaluación, siendo estas flexibles y abiertas, para favorecer una mayor participación y autonomía de los agentes implicados: la asignatura de EF (contenidos, objetivos, espacio educativo, recursos y materiales, contexto de la clase...); el profesor/a de EF (actitud hacia los alumnos/as, metodología y pedagogía, evaluación e imagen profesional...); el nivel 
de dificultad de la asignatura de EF (respecto a la calificación, respecto al nivel de exigencia, motivos que determinan el nivel de dificultad y consecuencias para el alumnado...); y la utilidad de la asignatura de EF (comparación temporal presente-futuro e importancia/estatus que recibe por parte de diferentes sectores: sistema educativo, centro docente, medios de comunicación, profesor/a de EF y de otras materias y las familias). Inductivamente a partir de los datos recogidos, fueron establecidas las categorías para el análisis y usadas en la presentación de los resultados.

\section{Criterios de credibilidad}

El criterio de credibilidad más destacable fue el de la triangulación de fuentes metodológicas (entrevistas, puzzle de Aronson y observación) y de personas (profesores, alumnos/as e investigadora). Por otro lado, se llevó a cabo una auditoría externa mediante la figura del "amigo crítico", como aconsejan Sparkes y Smith (2014), gracias a la colaboración de Daniel Martos García, Personal Docente e Investigador (PDI) titular de la Universitat de València (UV). Esta técnica permite "la ventaja de ser un punto de vista externo (...) la posibilidad de poder ver las debilidades que el propio investigador no ve" (Camarillo, 2011).

\section{Resultados y discusión}

\section{Objetivos de la EF y eficacia en su consecución}

De acuerdo con la investigación desarrollada por McEvoy et al (2014), los profesores A y B del presente trabajo coinciden en que el objetivo de la EF es preparar a la gente joven para que adopte un estilo de vida saludable basado en la práctica de actividad física. Los docentes consideran, que se basa en el "conocimiento de uno mismo, sus límites, sus valores, mejora de la auto-estima, descubrimiento del cuerpo (...) formarse a través del movimiento, (...) es una formación integral”. Podríamos afirmar que la EF persigue todos estos propósitos, tal y como Hardman (2011, citado por McEvoy, et al, 2014) clasificaba los objetivos curriculares de la EF alrededor del mundo, en orden de prevalencia como: 1) desarrollo de las habilidades motoras generales y específicas 2) promoción de la salud mediante estilos de vida saludables y activos y 3 ) desarrollo personal, social y moral. En primera instancia, los profesores A y $\mathrm{B}$, se refieren a los objetivos de la EF utilizando una definición estándar; sin embargo, al profundizar en el tipo de actividad docente (como se verá en el apartado correspondiente), apreciamos cómo el verdadero objetivo de la asignatura atiende al contexto y a la interpretación individual, difiriendo en función de las diferentes perspectivas que adquiere el valor de la actividad física (McEvoy, et al, 2014). Teniendo en cuenta esta pluralidad de percepciones individuales, puede parecer no tan importante el hecho de determinar un propósito compartido, tanto como establecer un acuerdo acerca de los límites que definen el comienzo y final de la EF (Kirk, 2009, citado por McEvoy, et al, 2014).

A pesar de ello, en términos generales, los objetivos tradicionales de la EF han estado centrados en conseguir resultados educativos y saludables, aunque la eficacia de la asignatura para estos propósitos ha estado cuestionada por Bailey et al (2009). A menor escala, esta investigación refuerza esta idea, ya que los grupos de alumnos/as A y B, coinciden en que "la EF es como una hora de descanso, no tienes que pensar, es moverte un poco y ya está". El grupo A, considera que sirve para "inflar la nota". No tienen trabajo diario, y si se les pide algo es una vez por trimestre, y no es muy costoso de hacer. El grupo de estudiantes B, afirma recibir una educación superficial, en la cual "se hace hincapié en principios teóricos del juego como la comunicación, el apoyo al compañero..., pero nunca se ponen en práctica y no se dedican clases para trabajar en ello". Añaden, que no persigue un objetivo de salud, ya que reconocen que las clases no son muy dinámicas: "no nos movemos". Ni siquiera consideran, que se trabaje la promoción de hábitos saludables: "en la unidad didáctica de condición física, no se trabajaron los hábitos saludables, por la metodología y las clases que planteó el profesor, que consistían en correr durante media hora". El grupo B concluye que "la EF es igual a competencia deportiva", y las clases están orientadas a "adquirir la habilidad técnica suficiente para jugar en equipo". 


\section{Estatus de la asignatura de EF y limitaciones del contexto}

McEvoy et al (2014) apuntaban que el contexto educativo ejerce una influencia sobre los puntos de vista de los participantes involucrados en el proceso de enseñanza-aprendizaje y sobre cómo estos puntos de vista, se trasladan a la práctica. Es más, insisten en la importancia de legitimar el lugar que ocupa la EF dentro las instituciones. En referencia a ello, todos los agentes participantes de esta investigación (alumnos/as y profesores/as), en general, consideran que la asignatura de EF es percibida como una asignatura poco importante, por parte de la Administración, del centro educativo, de las familias y de los profesores de otras materias. En este sentido, resulta interesante profundizar en la opinión de los protagonistas respecto a las limitaciones que favorecen este parecer generalizado.

La profesora A considera que la EF dentro del sistema educativo y a nivel de centro, se basa en una definición hipócrita, es decir, a nivel teórico sí se la valora, pero no se corresponde con la realidad del contexto del aula.

"Se están desdoblando las asignaturas instrumentales como lengua o matemáticas, mediante grupos de diversificación y contrato-programa que permiten trabajar de forma reducida, pero en EF doblan la ratio para sacar horas para estos grupos. Consideran más importante que los alumnos sean poquitos en matemáticas y les da igual que sean muchos en EF"

El profesor B coincide con esta definición y añade que es habitual escuchar entre las familias u otros profesores, comentarios como: “¿cómo gente con tan buenas notas ha suspendido EF? Los docentes consideran que la asignatura cuenta con una serie de limitaciones que hacen que el proceso educativo no sea como debiera y que la materia se perciba como una enseñanza no tan importante respecto a otras: el reducido número de horas semanales, el aumento de la ratio de alumnos, las instalaciones e incluso, la no actualización de su formación, la atribuyen a la poca calidad e interés de los cursos ofrecidos por el Estado.

Es evidente que se tratan de argumentos que dificultan la efectividad del proceso de enseñanzaaprendizaje, pero pueden existir además, otros condicionantes. Aludiendo a la diferenciación que hace Fernández-Balboa (2015) entre un conocimiento consciente y una reacción, podríamos ubicar a los docentes de este estudio dentro del segundo estado, ya que solo pueden reaccionar (pensar, sentir, hablar o actuar) frente a estímulos externos; sin embargo, no pueden ir más allá de las influencias ajenas y prestar atención a realidades personales más profundas. Este pensamiento se refuerza, si prestamos atención a las opiniones de ambos grupos de alumnos/as, ya que estos valoran positivamente el espacio educativo, a diferencia de los profesores. Es por ello, que el alumnado sitúa las limitaciones de la EF que reciben en el ejercicio profesional de los docentes, bien por aspectos metodológicos (el caso de la profesora A) o bien por la propia definición y aplicación pedagógica de la materia (el caso del profesor B).

\section{Ejercicio docente y limitaciones individuales}

El propósito de este apartado es profundizar en el tipo de actividad profesional de los docentes participantes en esta investigación, ya que a pesar de existir una concepción estándar teórica generalizada, el verdadero objetivo de la asignatura atiende a condicionantes del contexto (detallados en el apartado anterior según la percepción de los protagonistas) y a la interpretación individual sobre el valor de la actividad física. Es por ello, que en esta sección sí se ha hecho una distinción marcada entre los grupos A y B de alumnos/as y sus respectivos profesores, para poder aproximarnos con mayor objetividad a los dos perfiles profesionales de este estudio de caso, contrastando las percepciones de docentes y discentes.

Diversos estudios han mostrado que tener conocimiento de los contenidos y una buena pedagogía a la hora de mostrarlos, no garantiza la efectividad del profesor, ya que este además tiene que estar preparado para tratar con las emociones de los alumnos/as, así como con las dificultades fruto de las interacciones sociales que puedan suceder en el aula (Kiviniemi, 2000; Kontoniemi, 2003, citados por Klemola, et al, 2012; Pellicer, 2007). "La comprensión emocional es por lo tanto importante para la relación entre profesor-estudiante y el aprendizaje de la asignatura" (Klemola, et al, 2012, p.38). En este sentido, el grupo de la profesora A, afirma que esta tiene "muy buena actitud hacia sus alumnos/as, les apoya 
siempre y si tienes cualquier problema, aunque no sea de su asignatura te ayuda (...) es más cercana que los profesores de otras materias". La propia profesora A, reconoce que su actitud es variable dependiendo de factores como "el momento personal (...) o las características de los alumnos/as", pero en general destaca su proximidad hacia los estudiantes. Esto coincide con los resultados obtenidos por Koutrouba (2012), los cuales consideran que una relación de confianza entre profesor/a y alumnado, alienta a estos últimos a sentirse más cómodos y tener una mayor motivación hacia el aprendizaje, especialmente cuando el docente es comprensible, amable, flexible y utiliza herramientas de comunicación y socialización efectivas.

Sin embargo, tal y como comprobaron Klemola et al (2012), la promoción de un aprendizaje social y emocional no es sencillo y supone grandes dificultades como: la reacción frente a las emociones de los estudiantes en tareas de organización de la clase, el hecho de recordar las estrategias a utilizar e integrarlas de forma natural a la enseñanza y controlar las propias emociones del profesor/a cuando estos tratan con los alumnos/as. En este caso, la profesora A intenta llevar a cabo este tipo de metodología, pero en ciertos momentos se enfrenta a obstáculos que ella misma reconoce, como la necesidad de "tener la autoridad y que me respeten (...) y cuando he explicado lo que quiero conseguir, doy máxima libertad de participación". Esta sensación de pérdida de la autoridad en momentos puntuales, también es percibida por parte de sus alumnos/as, que piensan que en ocasiones "la profesora permite que haya alumnos/as que vayan a su bola, para que no molesten en la dinámica de la clase". Concuerda con Koutrouba (2012) en el hecho de que ser "efectivo", de ninguna manera significa ser maleable o demasiado flexible injustificadamente, sino por el contrario se asocia más con la firmeza y objetividad en las circunstancias que lo exijan.

Por otro lado, el grupo B considera que su profesor B tiene "una actitud muy negativa hacia los alumnos/as, hace correcciones negativas, pero no refuerza positivamente cuando lo haces bien”. Lo definen como de la "antigua escuela", llevando a cabo una metodología sin tener en cuenta lo que piensan sus alumnos/as u otros compañeros de profesión. El profesor B afirma no importarle reconocer que es "bastante seco, cortante (...) quiero que se trabaje en clase". Como vemos en este caso, no existe el intento de llevar a cabo una metodología emocional, ni existe consciencia del profesor acerca de su limitación en este sentido.

Teniendo en cuenta los resultados obtenidos por Koutrouba (2012), también se considera una enseñanza eficaz cuando los docentes tienden a involucrar a los estudiantes en procedimientos variados de aprendizaje y ponen en práctica técnicas de comunicación simple, comprensible, individualizada y participativa. Añaden además como orientaciones de éxito, cuando se preocupan por el conocimiento previo del alumnado, simplifican las tareas ajustándolas a las necesidades individuales, respetan la diversidad en cualquier ámbito y promueven metodologías solidarias y democráticas. Contrastando estos resultados con los del presente trabajo, podemos afirmar que ninguno de los dos perfiles se ajusta con precisión a las características descritas.

Por un lado, según el grupo A la profesora recurre a una programación negociada en la cual "no impone a los alumno/as las tareas", hecho que aprecian como algo positivo porque se sienten valorados; pero a su vez, reconocen que "improvisa y no se prepara las clases", haciendo que estas sean muy repetitivas. Habría que diferenciar por un lado, entre metodología democrática, donde a pesar de la participación de los alumnos/as, la autoridad la sigue teniendo el docente; y por otro lado, cuando son los discentes los que deciden qué se hace en cada momento. La docente A se excusa en llevar a cabo clases donde la técnica no sea tan importante para que "todos los alumnos/as puedan jugar", recurriendo con excesiva frecuencia a los juegos deportivos globales sin un planteamiento educativo específico, "jugar por jugar". Pero en el fondo, reconoce que la causa de planteamientos metodológicos tan pobres, se debe al proceso de acomodación sufrido y a la no preparación de las clases, en detrimento de la experiencia profesional adquirida. Sin embargo, este aspecto no influye negativamente en la motivación que los alumnos/as tienen hacia sus clases, ya que a pesar de que la asignatura no entusiasma a algunos por su poca utilidad, a la mayoría les agrada que la exigencia sea baja, se divierten mucho y la relación con la profesora es buena. Tiene mayor repercusión a la hora de calificar. La profesora A reconoce: 
"Odio la evaluación (...) me la invento a través del proceso (...) según el grupo o según el nivel, a mitad de trimestre estoy pensando qué es lo que voy a evaluar (...) en función de la clase y aprendizaje que están teniendo (...) Evalúo para que un grupo de alumnos/as puedan sacar un 10 y de ahí saco los criterios para que todo el mundo pueda aprobar"

Al no existir una programación real, ni una preparación de las clases, ni una metodología sólida, tampoco existen criterios de evaluación ni de calificación. Sus alumnos/as perciben esta limitación, y consideran que la profesora es excesivamente subjetiva y puntúa muy alto: "en ocasiones ha aprobado a gente que no se lo merecía (...) a algunas personas les exige más que a otras". Sin embargo, la profesora A para tener a sus alumnos/as satisfechos, pone el nivel de exigencia en un valor muy bajo, hecho que permite el aprobado de los discentes sin tener mucha dedicación. Paradójicamente, la profesora A escoge este tipo de evaluación para prestar mayor atención al esfuerzo realizado en clase y al proceso: "dedica al apartado de actitud hasta 3 puntos de la nota global", aunque no tiene criterios definidos para distribuir objetivamente esta puntuación; y "controla la asistencia de las clases llevando un control de las faltas y pidiendo justificantes por las ausencias", valorando como una actitud puntuable la mera asistencia al aula (cuando la asistencia a clase lo único que te garantiza es el derecho a evaluación continua).

Frente a esto, por un lado, algunos alumnos/as prefieren que "la evaluación sea subjetiva y no del gesto técnico", aunque parte de esta opinión se pueda ver influenciada por el evidente rechazo a la evaluación que hace el otro profesor de la asignatura y al desconocimiento de otras formas de medir el aprendizaje. Sin embargo, otra parte de los alumnos/as considera que evaluar el proceso no es "te mira $y$ lo que ella crea, te pone una nota", sino que tiene que estar sustentada por unos criterios de evaluación y calificación específicamente definidos.

Centrándonos en el caso del profesor $\mathrm{B}$, el origen del problema no está tanto en la metodología y pedagogía empleadas, sino en la propia definición de la asignatura:

"A partir de primero de Bachillerato (...) el deporte os va a costar dinero. Aquí se dan ideas de actividades que os pueden ayudar a que os salga gratis (...) si te dice tu pareja, vamos a bailar y no sabes, te costará dinero aprender (...) tiene una labor social también, si con 15 años puedes irte a la playa a jugar a voleibol y nadie te excluye porque no sepas jugar".

Por un lado, se aprecia cómo se hace referencia inconscientemente, a la promoción de actividad física como si esta fuera deporte; por otro lado, y consecuentemente, no la promociona por los beneficios intrínsecos de la primera, sino por los beneficios económicos asociados al negocio deportivo. Secundariamente, establece una relación entre socialización/inclusión y habilidad deportiva o rendimiento, que inconscientemente es la que transmite a sus alumnos/as. Así, el grupo B, considera que el profesor B "valora más a los chicos que a las chicas y a las personas que sabe que hacen deporte fuera del instituto". El propio profesor no es consciente de esta limitación y reconoce que "hay unas deficiencias que intentamos igualar (...) la experiencia deportiva previa del alumno/a determina muchísimo". Es más, a pesar de no exponerlo explícitamente, discrimina en función del sexo y del deporte practicado, clasifica a sus alumnos/as únicamente en función de si tienen más una habilidad orientada a "enseñanzas con balón" o a "enseñanzas rítmicas".

Abordando en la metodología empleada, el profesor B aplica el mando directo, es decir, "tiene el poder de la clase y obliga a sus alumnos/as a hacer las tareas sin reflexionar acerca del porqué o del proceso, o sin tener en cuenta sus preferencias o inquietudes". El propio profesor B reconoce que sus clases son una "especie de dictadura". En cuanto a la dinámica de las sesiones, el docente afirma utilizar una metodología "bastante más analítica de la que se puede considerar como ideal", es decir, traducido a la percepción de los discentes "las sesiones se repiten haciendo siempre lo mismo, practicar las técnicas y partido". En este sentido, el profesor B reconoce que improvisa en sus clases, pero no concibe esto como una limitación, apoyándose en los años de experiencia que avalan su actuación. Es más, reconoce que no prepararse las clases ha supuesto una mejora personal, ya que así consigues "evitar la frustración de plantear una clase perfecta en papel, y luego no corresponderse con la realidad". 
Frente a una definición de la EF igual a deporte, a una metodología exclusivamente analítica y a una distinción entre el alumnado en función del sexo y nivel deportivo previo, no es de extrañar que el tipo de evaluación que el profesor B considere como idónea, esté centrada en una "nota única de un partido o una serie de ejercicios técnicos muy concretos". Lo compara con "un examen de inglés, que te lo juegas delante del papel. Has estudiado muchas horas, has tenido ayuda y te lo juegas a una carta: sale bien o sale mal". El propio docente considera que este apartado es:

"uno de los caballos de batalla que tiene con los estudiantes (...) los alumnos/as piensan en pasárselo bien, jugar y hacer una actividad fisica muy cercana a lo recreativo. Yo intento tener una enseñanza de rendimiento (...) existe un conflicto de intereses"

Frente a esto, los discentes del grupo B consideran que los criterios de evaluación y calificación utilizados son "injustos y no valoran el esfuerzo" individual.

Antes de pasar a la conclusión, se resumen en la Tabla 3 los resultados obtenidos.

Tabla 3. Resumen de los resultados obtenidos

\begin{tabular}{|c|c|c|c|c|}
\hline & Alumnos/as A & Profesora A & Alumnos/as B & Profesor B \\
\hline $\begin{array}{l}\text { Objetivos de la } \\
\text { EF y eficacia en } \\
\text { su consecución }\end{array}$ & $\begin{array}{l}\text { Hora de descanso, no } \\
\text { hay que pensar, } \\
\text { moverse un poco, } \\
\text { infla la nota, no nos } \\
\text { movemos }\end{array}$ & $\begin{array}{l}\text { Auto-conocimiento, } \\
\text { valores, } \\
\text { descubrimiento } \\
\text { corporal, formación a } \\
\text { través del } \\
\text { movimiento, } \\
\text { formación integral }\end{array}$ & $\begin{array}{l}\text { Hora de descanso, } \\
\text { no hay que pensar, } \\
\text { moverse un poco, } \\
\text { igual a competencia } \\
\text { deportiva }\end{array}$ & $\begin{array}{l}\text { Auto-conocimiento, } \\
\text { valores, descubrimiento } \\
\text { corporal, formación a } \\
\text { través del movimiento, } \\
\text { formación integral }\end{array}$ \\
\hline $\begin{array}{c}\text { Estatus EF y } \\
\text { limitaciones del } \\
\text { contexto }\end{array}$ & - & $\begin{array}{c}\text { Planteamiento } \\
\text { hipócrita centro, ratio } \\
\text { desproporcionada } \\
\text { para priorizar } \\
\text { asignaturas } \\
\text { instrumentales }\end{array}$ & - & $\begin{array}{l}\text { Desprecio por parte de las } \\
\text { familias, reducido número } \\
\text { de horas semanales, } \\
\text { aumento de la ratio de } \\
\text { alumnos/as, formación de } \\
\text { actualización pobre }\end{array}$ \\
\hline $\begin{array}{c}\text { Ejercicio } \\
\text { docente y } \\
\text { limitaciones } \\
\text { individuales }\end{array}$ & $\begin{array}{l}\text { Buena actitud hacia } \\
\text { los alumnos/as, no } \\
\text { tiene control sobre } \\
\text { todos los alumnos/as } \\
\text { de la clase, } \\
\text { programación } \\
\text { negociada, } \\
\text { improvisación, poca } \\
\text { utilidad pero proceso } \\
\text { de aprendizaje } \\
\text { divertido, prefieren } \\
\text { evaluación subjetiva y } \\
\text { no técnica aunque les } \\
\text { gustaría que estuviera } \\
\text { respaldada por unos } \\
\text { criterios de } \\
\text { calificación }\end{array}$ & $\begin{array}{l}\text { Actitud cercana, } \\
\text { sensación de pérdida } \\
\text { de autoridad, } \\
\text { metodología } \\
\text { emocional con éxito } \\
\text { relativo, tiene auto- } \\
\text { consciencia de sus } \\
\text { limitaciones, priorizar } \\
\text { el juego a la técnica, } \\
\text { acomodo profesional, } \\
\text { no preparación de } \\
\text { clases, evaluación y } \\
\text { calificación } \\
\text { improvisada, primar } \\
\text { el esfuerzo y el } \\
\text { proceso, evaluación } \\
\text { subjetiva }\end{array}$ & $\begin{array}{c}\text { Actitud muy } \\
\text { negativa, poco } \\
\text { feedback y negativo, } \\
\text { valora más a los } \\
\text { chicos y a las chicas } \\
\text { deportistas, no hay } \\
\text { reflexión de las } \\
\text { tareas, no tiene en } \\
\text { cuenta nuestras } \\
\text { inquietudes, sesiones } \\
\text { repetitivas, criterios } \\
\text { de evaluación y } \\
\text { calificación injustos } \\
\text { y no orientados al } \\
\text { proceso y esfuerzo }\end{array}$ & $\begin{array}{l}\text { Actitud distante, búsqueda } \\
\text { de rendimiento, } \\
\text { concepción consumista y } \\
\text { elitista, EF=Deporte, no } \\
\text { tiene auto-consciencia de } \\
\text { sus limitaciones como } \\
\text { profesor, discrimina por } \\
\text { sexo y nivel deportivo, } \\
\text { mando directo, analítico, } \\
\text { improvisación justificada } \\
\text { en sus años de experiencia, } \\
\text { evaluación y calificación } \\
\text { técnica y de resultado }\end{array}$ \\
\hline
\end{tabular}




\section{Conclusiones}

A razón del análisis de los resultados obtenidos, podemos afirmar que a pesar de que los profesores/as parecen tener muy clara una definición teórica estandarizada acerca del propósito de la asignatura de EF, la realidad en la aplicación práctica del aula es muy distinta. Para poder comprender el porqué de la poca eficacia de la asignatura en la consecución de sus objetivos, se ha prestado atención a las limitaciones relacionadas con el contexto y el estatus que la materia adquiere en él; y por otro lado, a pesar de que los docentes protagonistas no son conscientes de ellas, a las limitaciones individuales del ejercicio profesional que adoptan. En el caso de la profesora A, limitaciones enfocadas en la metodología y recursos pedagógicos empleados y en el caso del profesor $\mathrm{B}$, partiendo desde el propio planteamiento inicial del concepto de la EF.

En vista de la variabilidad de perspectivas que adopta la EF (incluso dentro del departamento educativo de un mismo centro), podemos afirmar con certeza que en futuras líneas de investigación resultaría interesante determinar los límites que definen el comienzo y el final de la materia, antes que intentar encontrar cuál es el propósito universal de esta. Así, como sería interesante promover estrategias de cooperación entre los docentes dentro de un mismo departamento, como herramientas de formación continua, auto-reflexión y auto-crítica profesional y toma de consciencia tanto de las limitaciones contextuales como de las individuales.

Por otro lado, resaltar los aspectos positivos de la evaluación o aproximación al proceso de enseñanzaaprendizaje empleando la metodología competencial del AC y específicamente, la técnica del Puzzle de Aronson. El trabajo de reflexión y crítica en grupos pequeños heterogéneos y la co-responsabilidad de los agentes participantes, ha permitido llevar a cabo una evaluación en profundidad, objetiva y rigurosa, mediante la discusión y contraste de las percepciones de los diferentes roles (profesorado, alumnado, investigadora). Por limitaciones temporales, no se ha podido llevar a cabo, pero hubiera sido interesante elaborar un informe con los resultados, para que alumnos/as y profesorado, los discutiera y cooperaran para llevar a cabo las pertinentes adaptaciones del proceso de enseñanza-aprendizaje. Así, el proyecto hubiera ido más allá de la reflexión y hubiera podido tener una aplicación práctica posterior. A pesar de no haberse podido completar, el simple proceso de reflexión ha supuesto una tarea formativa de responsabilidad, autonomía y sentido crítico para todos los participantes.

\section{Bibliografía}

Bailey, R., Armour, K., Kirk, D., Jess, M., Pickup., I, Sandford, R. \& BERA Physical Education and Sport Pedagogy Special Interest Group. (2009). The educational benefits claimed for physical education and school sport: An academic review. Research Papers in Education, 24(1), 1-27.

Camarillo, G. C. (2011, December 12). Confiabilidad y validez en estudios cualitativos. Revista "Educación y Ciencia," 1(15).

Fernández-Balboa, J. M. (2015). Imploding the boundaries of transformative/critical pedagogy and research in physical education and sport pedagogy: Looking inward for (self-)consciousness/knowledge and transformation. Sport, Education and Society, DOI: 10.1080/13573322.2015.1050371

Goetz, J., \& LeCompte, M. (1988). Etnografía y diseño cualitativo en investigación educativa. Madrid: Morata.

Gutiérrez Sanmartín, M., \& Pilsa Doménech, C. (2006). Actitudes de los alumnos hacia la Educación Física y sus profesores. Revista Internacional de Medicina y Ciencias de la Actividad Física y el Deporte vol. 6 (24) pp. 212-229

Gutiérrez, M., \& López, E. (2012). Motivación, comportamiento de los alumnos y rendimiento académico. Infancia y Aprendizaje: Journal for the Study of Education and Development, 35:1, 61-72

Hodges Kulinna, P., Cothran, D. J., \& Regualos, R. (2006). Teachers' reports of student misbehavior in physical education. Research Quarterly for Exercise and Sport, 77:1, 32-40 
Huéscar, E., \& Moreno-Murcia, J. A. (2012). Relación del tipo de feed-back del docente con la percepción de autonomía del alumnado en clases de educación física. Infancia y Aprendizaje: Journal for the Study of Education and Development, 35:1, 87-98

Klemola, U., Heikinaro-Johansson, P., \& O'Sullivan, M. (2013). Physical education student teachers' perceptions of applying knowledge and skills about emotional understanding studied in PETE in a oneyear teaching practicum. Physical Education and Sport Pedagogy, 18:1, 28-41, DOI: 10.1080/17408989.2011.630999

Koutrouba, K. (2012). A profile of the effective teacher: Greek secondary education teachers' perceptions. European Journal of Teacher Education, 35:3, 359-374, DOI: $10.1080 / 02619768.2011 .654332$

Kvale, S. (2011). Las entrevistas en la investigación cualitativa. Madrid: Morata.

Martínez, J., \& Gómez, F. (2010). La técnica puzzle de Aronson: Descripción y desarrollo. En Arnaiz, P.; Hurtado, Ma.D. y Soto, F.J. (Coords.) 25 Años de Integración Escolar en España: Tecnología e Inclusión en el ámbito educativo, laboral y comunitario. Murcia: Consejería de Educación, Formación y Empleo.

McEvoy, E., Heikinaro-Johansson, P., \& MacPhail, A. (2015). Physical education teacher educators' views regarding the purpose(s) of school physical education. Sport, Education and Society, DOI: 10.1080/13573322.2015.1075971

Moreno, J. A., \& Hellín, M. G. (2007). El interés del alumnado de Educación Secundaria Obligatoria hacia la Educación Física. Revista Electrónica de Investigación Educativa, 9 (2).

Pellicer, I (2007). Educación física emocional. Postgrau en Educació Emocional i Benestar, Facultat de Pedagogia, Departament de Mètodes d'Investigació i Diagnòstic en Educació, Universitat de Barcelona, curs: 2006-2007, Tutora: Ma Pilar Lozano

Rink, J. E. (2013). Measuring teacher effectiveness in physical education. Research Quarterly for Exercise and Sport, 84:4, 407-418, DOI: 10.1080/02701367.2013.844018

Rodríguez, P. L., García-Cantó, E., Sánchez-López, C., \& López-Miñarro, P. A. (2013). Percepción de la utilidad de las clases de educación física y su relación con la práctica físico-deportiva en escolares. Cultura y Educación: Culture and Education, 25:1, 65-76, DOI: 10.1174/113564013806309127

Shoval, E., Erlich, I., \& Fejgin, N. (2010). Mapping and interpreting novice physical education teachers' self-perceptions of strengths and difficulties. Physical Education and Sport Pedagogy, 15:1, 85101, DOI: 10.1080/17408980902731350

Sparkes \& Smith (2014). Qualitative reserach Methods In Sport, Exercise and Health. From process to product. London: Routledge

Stake, R.E. (4 ${ }^{\mathrm{a}}$ edición) (2007). Investigación con estudio de casos. Madrid: Morata

Velazquez, C., Fraile, A., \& Lopez Pastor, V. (2014). Aprendizaje cooperativo en educación física. Movimiento, 20 (1), 239-259.

Wolcott, H. (2003). The man in the principal's office: An Ethnography. Altamira Press

\section{Legislación}

España. Real Decreto 1105/2014, de 26 de diciembre, por el que se establece el currículo básico de la Educación Secundaria Obligatoria y del Bachillerato, Boletín Oficial del Estado (BOE), num. 3, 3 de enero de 2015

España. Ley Orgánica 8/2013, de 9 de diciembre, para la Mejora de la Calidad Educativa (LOMCE ), Boletín Oficial del Estado (BOE ), num. 295, 10 de diciembre de 2013

España. Ley Orgánica 2/2006, de 3 de mayo, de Educación (LOE), Boletín Oficial del Estado (BOE), num. 106, de 4 de mayo de 2006 